\title{
SIMULAÇÃO MONTE CARLO NO ENSINO DE LUMINESCÊNCIA E CINÉTICA DE DECAIMENTO DE ESTADOS EXCITADOS
}

\author{
Herbert Winnischofer, Márcio Peres de Araújo, Lauro Camargo Dias Júnior e João Batista Marques Novo* \\ Departamento de Química, Universidade Federal do Paraná, CP 19081, 81531-990 Curitiba - PR, Brasil
}

Recebido em 16/12/08; aceito em 22/6/09; publicado na web em 27/11/09

\begin{abstract}
MONTE CARLO SIMULATION ON TEACHING OF LUMINESCENCE AND EXCITED STATES DECAY KINETICS. A software based in the Monte Carlo method have been developed aiming the teaching of important cases of mechanisms found in luminescence and in excited states decay kinetics, including: multiple decays, consecutive decays and coupled systems decays. The Monte Carlo Method allows the student to easily simulate and visualize the luminescence mechanisms, focusing on the probabilities of the related steps. The software CINESTEX was written for FreeBASIC compiler; it assumes first-order kinetics and any number of excited states, where the pathways are allowed with probabilities assigned by the user.
\end{abstract}

Keywords: Monte Carlo simulation; luminescence; excited states decay kinetics.

\section{INTRODUÇÃO}

Os processos de emissão de luz estão presentes em diversos fenômenos naturais e são importantes nas interações do Homem com o meio ambiente. Existem basicamente dois modos pelos quais as substâncias podem emitir luz sob condições normais. O primeiro é por intermédio de aquecimento e é chamado de incandescência. A luz emitida neste caso segue o perfil da radiação de corpo negro e é dependente da temperatura. O segundo modo é denominado luminescência, que é responsável pela emissão de luz sob temperaturas relativamente mais baixas. Esta luz emitida é característica da amostra, pois depende da existência de estados eletrônicos (excitados e fundamental) característicos da substância.

A luminescência pode ser classificada segundo o tipo de fonte de excitação: ${ }^{1}$

- fotoluminescência refere-se à excitação por meio de fótons (luz). Exemplos deste tipo de emissão são encontrados em botões de interruptores de luz, sinalizadores de emergência etc;

- catodo-luminescência quando a excitação for realizada com raios catódicos (feixes de elétrons). São encontrados nos tubos de raios catódicos (CRT) de televisores e monitores de computadores;

- eletroluminescência quando a excitação for realizada por meio de corrente elétrica. São encontrados em diodos emissores de luz (Light Emitting Diodes, LED), como luzes indicadoras em aparelhos eletrônicos de som, vídeo etc;

- quimiluminescência quando a fonte de excitação for um reagente químico no estado eletrônico excitado. Como exemplo tem-se a reação do luminol, que é empregado em investigações criminais;

- bioluminescência quando o reagente químico responsável pela luminescência fizer parte do metabolismo de um ser vivo. Exemplos deste tipo são encontrados em vaga-lumes, algas, peixes abissais etc; termo-luminescência quando a luminescência for estimulada por meio de aquecimento da amostra (se contrapõe à incandescência devido à emissão de luz característica da amostra).

Outro tipo de classificação considera o tempo de duração da emissão de luz. Temos assim: fluorescência, quando a emissão de luz terminar quando cessar a excitação da amostra e, fosforescência, quando a emissão de luz perdurar por um período de tempo considerável após findar a excitação da amostra.

*e-mail: jbmnovo@quimica.ufpr.br
O entendimento dos processos envolvidos nestes fenômenos passa, muitas vezes, pela análise dos mecanismos cinéticos das populações dos estados eletrônicos excitados da amostra. Entender tais comportamentos cinéticos pode ser difícil e trabalhoso, pois muitas vezes estes processos apresentam equações matemáticas muito complexas. Neste sentido, o método Monte Carlo é extremamente útil, pois permite que se simule sistemas complexos de modo muito simples, sem que seja necessário saber, a priori, qual é a equação cinética associada. A denominação "Método Monte Carlo"2 tornouse uma expressão geral associada ao uso de números aleatórios e de estatística de probabilidade. Para que a simulação Monte Carlo esteja presente em um estudo, basta que este faça uso de números aleatórios na verificação de algum problema. ${ }^{2}$

Neste trabalho, são apresentados vários casos de comportamentos cinéticos importantes encontrados em luminescência e em cinética de estados excitados, que podem ser estudados sem a necessidade do conhecimento das equações analíticas associadas.

\section{O PROGRAMA E O MÉTODO DE SORTEIO}

O programa de simulação Monte Carlo implementado neste trabalho (CINESTEX) pode ser utilizado para simulação de processos em cinética de estados excitados e também em cinética química, e possui as seguintes características: 1) quaisquer números de estados excitados (ou reagentes) podem ser considerados; 2) todos os caminhos de conversão entre os $\mathrm{N}$ estados (ou reagentes) são possíveis, e 3) atribuição de probabilidade de conversão para cada caminho existente.

O método de sorteio emprega o modelo de urna de Ehrenfest ${ }^{3}$ e é realizado do seguinte modo: 1) sorteia-se uma determinada molécula de um estado X; 2) se a molécula existir neste estado (ou seja, se ainda não sofreu conversão de estado), sorteia-se um dos $\mathrm{N}^{2}$ caminhos possíveis, de acordo com as probabilidades de conversão atribuídas previamente pelo usuário; 3) se o caminho sorteado $\mathrm{X} \rightarrow \mathrm{Y}$ estiver disponível, a molécula sorteada irá para o estado Y, caso contrário, permanece em $X$.

\section{RESULTADOS E DISCUSSÃO}

Apresentamos, a seguir, alguns tipos de simulação de importância no estudo de luminescência e de cinética de estados excitados. 


\section{Decaimentos múltiplos}

A Figura 1a apresenta o diagrama para dois decaimentos, a partir do mesmo estado excitado. Este tipo de mecanismo pode ser encontrado no caso de íons $\mathrm{Eu}^{3+}$, onde o decaimento $1 \rightarrow 2$, com probabilidade $\mathrm{k}_{12}$, é devido a uma transição eletrônica de alta intensidade $\left({ }^{5} \mathrm{D}_{0} \rightarrow{ }^{7} \mathrm{~F}_{2}\right)$ e o decaimento $1 \rightarrow 3$, com probabilidade $\mathrm{k}_{13}$, é devido a uma transição eletrônica de baixa intensidade $\left({ }^{5} \mathrm{D}_{0} \rightarrow{ }^{7} \mathrm{~F}_{1}\right)$. Na Figura 2 temos a simulação Monte Carlo para esse diagrama, onde a curva de decaimento do estado emissor 1 é coincidente com a curva cuja probabilidade $\mathrm{k}$ é a soma das probabilidades das transições $\mathrm{k}_{12} \mathrm{e} \mathrm{k}_{13}$, ou seja, $\mathrm{k}=\mathrm{k}_{12}+\mathrm{k}_{13}$. Consequentemente, como os dois decaimentos são função da população do mesmo estado excitado 1, conclui-se que as duas transições, $1 \rightarrow 2$ e $1 \rightarrow 3$, terão o mesmo decaimento, dado pela probabilidade total $\mathrm{k}=\mathrm{k}_{12}+\mathrm{k}_{13}$. Sendo assim, não é possível obter as probabilidades individuais $\mathrm{k}_{12} \mathrm{e}_{13}$, por meio das curvas de decaimento das duas emissões, mas apenas a probabilidade total $\mathrm{k}_{12}+\mathrm{k}_{13}$. Deve-se mencionar que, apesar destas transições serem monitoradas pelo espectrômetro em diferentes comprimentos de onda de emissão e de terem diferentes probabilidades de emissão, ainda assim o decaimento do estado 1 seguirá a probabilidade total $\mathrm{k}_{12}+\mathrm{k}_{13}$.

A equação diferencial para este tipo de sistema pode ser descrita por:

$\mathrm{dN} 1 / \mathrm{dt}=-\left(\mathrm{k}_{12}+\mathrm{k}_{13}\right) \cdot \mathrm{N} 1$

onde N1 é a população (ou concentração) do estado excitado 1. A solução para esta equação é um decaimento exponencial simples, da forma:

$\mathrm{N} 1(\mathrm{t})=\mathrm{N} 1(0) \cdot \exp \left[-\left(\mathrm{k}_{12}+\mathrm{k}_{13}\right) \mathrm{t}\right]$

onde N1(0) é a população do estado excitado 1 no tempo inicial, $\mathrm{t}=0$. Note-se que o decaimento exponencial é função da soma das duas probabilidades $\left(\mathrm{k}_{12}+\mathrm{k}_{13}\right)$, concordando com a simulação Monte Carlo.

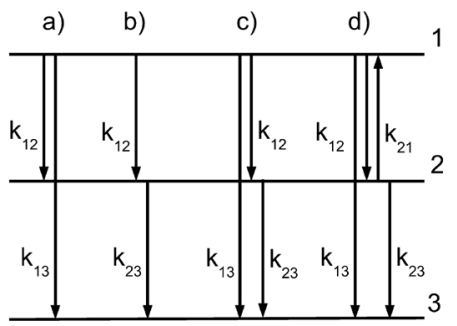

Figura 1. Esquema de decaimentos (a) múltiplos com probabilidades de transições $k_{12}$ e $k_{13}$, (b) consecutivos com probabilidades de emissão $k_{12}$ e $k_{23}$, (c) consecutivos com emissão dos estados 1 e 2, dadas pelas probabilidades $k_{13}$ e $k_{23}$ e (d) de dois estados excitados acoplados por meio de transições $1 \leftrightarrows 2$, exibindo emissões $1 \rightarrow 3$ e $2 \rightarrow 3$, dadas pelas probabilidades $k_{13}$ e $k_{23}$, respectivamente

\section{Decaimentos consecutivos}

A Figura $1 \mathrm{~b}$ apresenta o diagrama para decaimentos consecutivos $1 \rightarrow 2 \rightarrow 3$ de dois estados excitados ( 1 e 2 ). As equações diferenciais para as populações dos estados excitados 1 e 2 são:

$\mathrm{dN} 1 / \mathrm{dt}=-\mathrm{k}_{12} \cdot \mathrm{N} 1$

$\mathrm{dN} 2 / \mathrm{dt}=\mathrm{k}_{12} \cdot \mathrm{N} 1-\mathrm{k}_{23} \cdot \mathrm{N} 2$

As soluções para este sistema de equações são: ${ }^{4}$

$\mathrm{N} 1(\mathrm{t})=\mathrm{N} 1(0) \cdot \exp \left(-\mathrm{k}_{12} \cdot \mathrm{t}\right)$

$$
\mathrm{N} 2(\mathrm{t})=\mathrm{P} \cdot\left[\exp \left(-\mathrm{k}_{12} \cdot \mathrm{t}\right)-\exp \left(-\mathrm{k}_{23} \cdot \mathrm{t}\right)\right]+\mathrm{N} 2(0) \cdot \exp \left(-\mathrm{k}_{23} \cdot \mathrm{t}\right)
$$

onde $\mathrm{P}=\mathrm{k}_{12} \cdot \mathrm{N} 1(0) /\left(\mathrm{k}_{23}-\mathrm{k}_{12}\right)$. Note que, neste sistema, N1 segue o decaimento exponencial simples, enquanto que $\mathrm{N} 2$ inicia em zero (se $\mathrm{N} 2(0)=0)$, passa por um máximo e depois decai a zero.

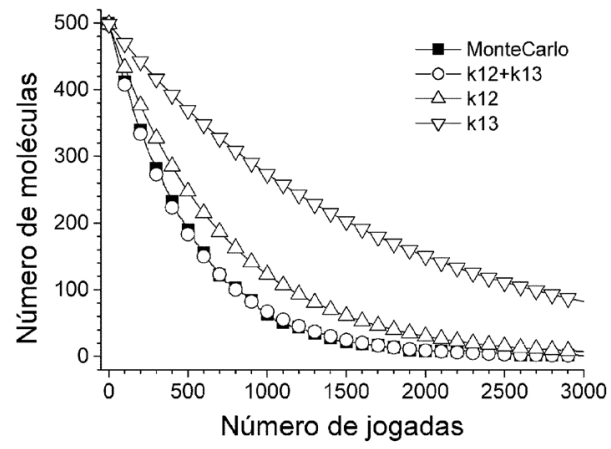

Figura 2. Curvas associadas aos decaimentos múltiplos $1 \rightarrow 2$ e $1 \rightarrow 3$, com probabilidades de emissão $k_{12}$ e $k_{13}$, respectivamente. São também mostrados $o$ decaimento com probabilidade de emissão $k_{12}+k_{13}$ e o decaimento simulado pelo método Monte Carlo (com 3 estados, 500 moléculas no estado 1, $k_{12}=0,70$ e $k_{13}=0,30$ )

Este mecanismo apresenta dois casos limites importantes:

$1^{\circ}$ caso: $k_{12}>k_{23}$. Este caso ocorre em moléculas que foram excitadas em estados singletes superiores (Estado $1=\mathrm{S}_{2}, \mathrm{~S}_{3}$, etc.) em relação ao estado singlete emissor (Estado $2=\mathrm{S}_{1}$ ). A relaxação dos níveis superiores até o nível emissor é muito rápida (etapa $1 \rightarrow 2$, que é da ordem de picossegundos) em comparação com o decaimento fluorescente (etapa $2 \rightarrow 3$, que é da ordem de nanossegundos). Observa-se, neste caso, que o perfil do decaimento do estado emissor $\mathrm{S}_{1}$ segue o do decaimento fluorescente (que é a etapa lenta $2 \rightarrow 3$ ), como se ele tivesse sido excitado diretamente. A Figura 3 apresenta a simulação Monte Carlo para esta situação. Observa-se que o decaimento do estado superior (N1) apresenta um decaimento muito rápido, acompanhado por uma ascensão rápida da curva do estado 2 (N2). Este rápido transiente é devido à alta probabilidade de transição $1 \rightarrow 2 \mathrm{em}$ relação à probabilidade da transição $2 \rightarrow 3$. No limite onde $k_{12}>>$ $\mathrm{k}_{23}$, teríamos este transiente inicial sendo praticamente instantâneo e o estado 2 apresentaria uma curva de decaimento (N2) parecendo com uma exponencial simples, como se ele tivesse sido excitado diretamente. A equação para a população do estado 2 seria dependente da etapa lenta $\left(\mathrm{k}_{23}\right)$ para tempos longos, após o transiente inicial: ${ }^{4}$

$\mathrm{N} 2(\mathrm{t})=\mathrm{N} 1(0) \cdot \exp \left(-\mathrm{k}_{23} \cdot \mathrm{t}\right)$

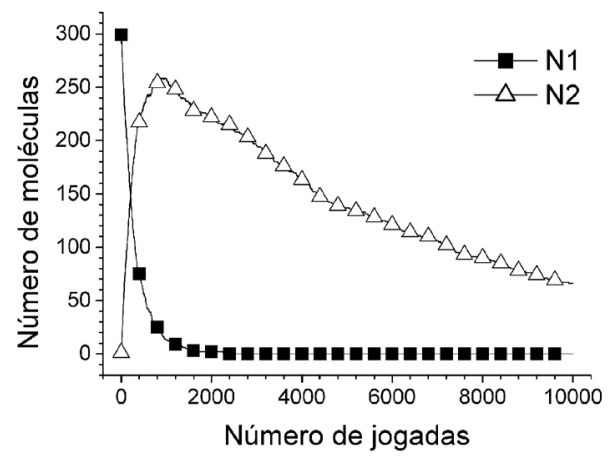

Figura 3. Curvas dos estados 1 e 2, associadas aos decaimentos consecutivos $1 \rightarrow 2 \rightarrow 3$ no limite $k_{12}>k_{23}$. A simulação foi efetuada com 3 estados, 300 moléculas no estado 1 e probabilidades $k_{12}=20$ e $k_{23}=1$ 
$2^{\circ}$ caso: $\mathrm{k}_{12}<\mathrm{k}_{23}$. Este caso ocorre em situações onde moléculas que apresentam decaimento fluorescente muito rápido têm seus decaimentos obtidos por um espectrômetro de resposta lenta, ou seja, que apresenta largura temporal do pulso de excitação mais larga que a emissão da amostra. A fonte de excitação comporta-se como um "estado excitado de tempo de vida longo". Neste caso, a curva de decaimento registrada no espectrômetro segue o perfil do pulso de excitação, e não se consegue registrar o perfil verdadeiro do decaimento rápido da emissão da amostra. A Figura 4 apresenta a simulação Monte Carlo para esta situação. Observa-se que a curva de decaimento da fluorescência do estado 2 (N2) segue o perfil de decaimento lento do pulso de excitação (curva $\mathrm{N} 1$, com $\mathrm{k}_{12}=1$ ) e, deste modo, não se consegue obter o perfil de decaimento verdadeiro ( $\operatorname{com~k}_{23}=4$ ).

A equação para a população do estado 2 seria dependente da etapa lenta $\left(\mathrm{k}_{12}\right)$ para tempos longos, após o transiente inicial: ${ }^{4}$

$\mathrm{N} 2(\mathrm{t})=\left[\mathrm{k}_{12} \cdot \mathrm{N} 1(0) / \mathrm{k}_{23}\right] \cdot \exp \left(-\mathrm{k}_{12} \cdot \mathrm{t}\right)$

ou seja, é proporcional a $\exp \left(-\mathrm{k}_{12} . \mathrm{t}\right)$. O decaimento segue o perfil da fonte de excitação e assim não é possível se obter $\mathrm{k}_{23}$, a não ser por meio de métodos matemáticos envolvendo convolução e deconvolução de curvas.

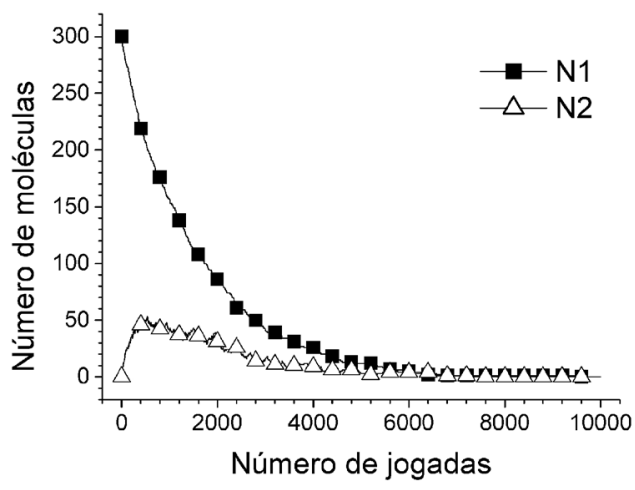

Figura 4. Curvas dos estados 1 e 2, associadas aos decaimentos consecutivos $1 \rightarrow 2 \rightarrow 3$ no limite $k_{12}<k_{23}$. A simulação foi efetuada com 3 estados, 300 moléculas no estado 1 e probabilidades $k_{12}=1$ e $k_{23}=4$

$3^{\circ}$ caso: um terceiro caso pode ser visto na Figura 1c. Esta Figura traz o diagrama para decaimentos consecutivos $1 \rightarrow 2 \rightarrow 3$ de dois estados excitados ( 1 e 2 ) que apresentam emissões dadas pelas probabilidades $\mathrm{k}_{13}$ e $\mathrm{k}_{23}$ e serve para ilustrar os processos de fluorescência (transição $1 \rightarrow 3$ ) e fosforescência (transição $2 \rightarrow 3$ ). Neste caso, o estado 1 é o estado singlete excitado $\left(\mathrm{S}_{1}\right)$, o estado 2 é o estado triplete $\mathrm{T}_{1}$ e o estado 3 é o estado singlete fundamental $\left(\mathrm{S}_{0}\right)$. O caminho $1 \rightarrow 2$ inclui o cruzamento inter-sistema. A Figura 5 mostra a simulação Monte Carlo para este exemplo. Note que a probabilidade de emissão $\mathrm{k}_{13}$ do estado $1\left(\mathrm{~N} 1=\mathrm{S}_{1}\right)$ é dez vezes maior que a probabilidade de emissão $\mathrm{k}_{23}$ do estado $2\left(\mathrm{~N} 2=\mathrm{T}_{1}\right)$ e, portanto, $\mathrm{o}$ decaimento fluorescente do estado $\mathrm{S} 1$ (curva N1) é bem mais rápido (da ordem de nanossegundos) que o decaimento fosforescente do estado T1 (curva N2, geralmente maior que microssegundos).

\section{Sistemas acoplados}

A Figura 1d apresenta o diagrama para dois estados excitados ( 1 e 2 ) acoplados por meio de transições $1 \leftrightarrows 2$, exibindo emissões $1 \rightarrow 3$ e $2 \rightarrow 3$, dadas pelas probabilidades $\mathrm{k}_{13}$ e $\mathrm{k}_{23}$, respectivamente. Este é um esquema similar ao dos decaimentos consecutivos, exceto que o estado 2 pode retro-alimentar o estado 1 . As equações diferenciais para este sistema são:

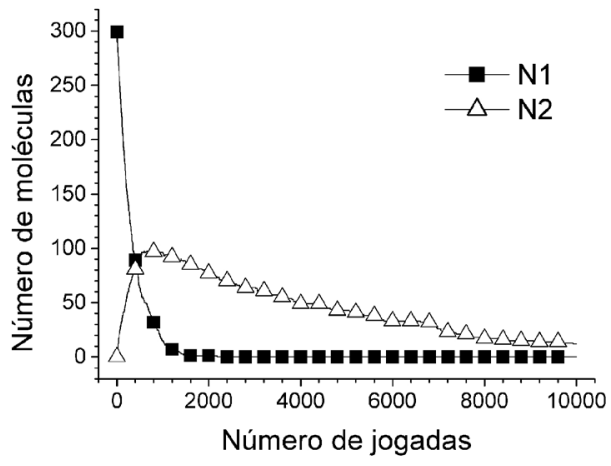

Figura 5. Curvas associadas aos decaimentos consecutivos $1 \rightarrow 2 \rightarrow 3$, exemplificando os processos de fluorescência (curva N1, transição $1 \rightarrow 3 \mathrm{com}$ probabilidade $k_{13}$ ) e fosforescência (curva $N 2$, transição $2 \rightarrow 3$ com probabilidade $k_{23}$ ). A simulação foi efetuada com 3 estados, 300 moléculas no estado $1, k_{12}=0,5, k_{13}=1$ e $k_{23}=0,1$

$\mathrm{dN} 1 / \mathrm{dt}=-\left(\mathrm{k}_{12}+\mathrm{k}_{13}\right) \cdot \mathrm{N} 1+\mathrm{k}_{21} \cdot \mathrm{N} 2$

$\mathrm{N} 2 / \mathrm{dt}=+\mathrm{k}_{12} \cdot \mathrm{N} 1-\left(\mathrm{k}_{21}+\mathrm{k}_{23}\right) \cdot \mathrm{N} 2$

Admitindo-se as condições de contorno no tempo zero, $\mathrm{N} 1=$ $\mathrm{N} 1(0)$ e N2 = N2(0) = 0, a essas equações diferenciais homogêneas de $1^{\text {a }}$ ordem, chega-se às soluções: ${ }^{4}$

$N 1(t)=N 1(0) \cdot\left[g_{1} \cdot \exp \left(-\gamma_{1} t\right)-g_{2} \cdot \exp \left(-\gamma_{2} t\right)\right]$

$\mathrm{N} 2(\mathrm{t})=\mathrm{N} 1(0) \cdot\left[\mathrm{k}_{12} /\left(\gamma_{1}-\gamma_{2}\right)\right] \cdot\left[\exp \left(-\gamma_{2} \mathrm{t}\right)-\exp \left(-\gamma_{1} \mathrm{t}\right)\right]$

onde: $\mathrm{g}_{1}=\left(\mathrm{X}-\gamma_{2}\right) /\left(\gamma_{1}-\gamma_{2}\right) ; \mathrm{g}_{2}=\left(\mathrm{X}-\gamma_{1}\right) /\left(\gamma_{1}-\gamma_{2}\right) ; \gamma_{1}=\{(\mathrm{X}+\mathrm{Y})$ $\left.+\left[(\mathrm{X}-\mathrm{Y})^{2}+4 \mathrm{k}_{12} \mathrm{k}_{21}\right]^{1 / 2}\right\} / 2 ; \gamma_{2}=\left\{(\mathrm{X}+\mathrm{Y})-\left[(\mathrm{X}-\mathrm{Y})^{2}+4 \mathrm{k}_{12} \mathrm{k}_{21}\right]^{1 / 2}\right\} / 2$; $\mathrm{X}=\mathrm{k}_{12}+\mathrm{k}_{13} ; \mathrm{Y}=\mathrm{k}_{21}+\mathrm{k}_{23}$

Neste caso, os estados 1 e 2 não decaem exponencialmente e exibem cinética de decaimento complexa: as duas curvas são somas de duas exponenciais com diferentes fatores pré-exponenciais, mas as probabilidades $\gamma_{1}$ e $\gamma_{2}$ para os termos exponenciais são os mesmos para as populações destes dois estados.

Este diagrama permite mostrar dois casos importantes: fluorescência atrasada e formação de excímeros.

$1^{\circ}$ caso: fluorescência atrasada ativada termicamente (tipo E). Vimos, no exemplo anterior, que o decaimento fluorescente é bem mais rápido, em relação ao decaimento fosforescente. Entretanto, algumas moléculas apresentam a emissão fluorescente do estado $\mathrm{S}_{1}$ com decaimento tão lento quanto o decaimento fosforescente do estado $\mathrm{T}_{1}$, fenômeno que se chama fluorescência atrasada. Este fenômeno é apresentado pela benzofenona ${ }^{5}$ e pode ser representado pelo caminho $1 \rightarrow 2 \rightarrow 1 \rightarrow 3$ no diagrama da Figura 1d, ou seja, pela re-população térmica do estado singlete $S_{1}$ (estado 1), via $T_{1}$ (estado 2). Neste caso, os estados 1 e 2 estão próximos em energia e as probabilidades de transição $1 \rightarrow 2$ e $2 \rightarrow 1$ são comparáveis entre si, o que faz com que se crie um estado de pseudo-equilíbrio dinâmico entre os estados 1 e 2, ou seja, a troca entre estados 1 e 2 é mais rápida que os decaimentos destes estados. A Figura 6 simula este estado de equilíbrio dinâmico $1 \leftrightarrows 2$. Pode-se observar nesta Figura, que, após um breve transiente inicial, as emissões dos dois estados $\left(\mathrm{N} 1=\mathrm{S}_{1}\right.$ e $\left.\mathrm{N} 2=\mathrm{T}_{1}\right)$ apresentam o mesmo decaimento exponencial longo. Este é o fenômeno de fluorescência atrasada que faz com que a fluorescência acabe apresentando um decaimento lento, graças ao acoplamento com o estado 2 (estado triplete $\mathrm{T}_{1}$ ), que age, neste caso, como um "armazenador de energia". 


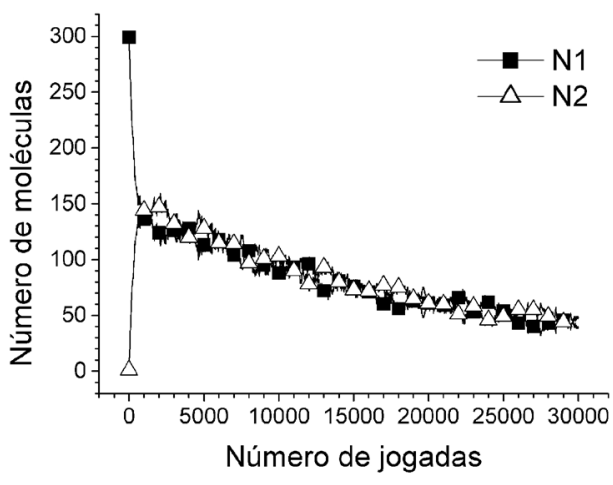

Figura 6. Curvas associadas ao sistema de dois estados excitados acoplados, exemplificando o processo de fluorescência atrasada. A simulação foi efetuada com 3 estados, 300 moléculas no estado 1 e probabilidades $k_{12}=1$, $k_{13}=0,05, k_{21}=1$ e $k_{23}=0,001$

$2^{\circ}$ caso: formação de excímeros. Os excímeros são dímeros de estado excitado, sendo que sua formação pode ser representada pela Equação 13:

$\mathrm{M}^{*}+\mathrm{M} \leftrightarrows(\mathrm{MM})^{*}$

onde * se refere a estado excitado.

O processo é bi-molecular, mas sob condições de baixa concentração de $\mathbf{M}^{*}$ (como, por exemplo, com fonte de excitação de baixa intensidade, onde $\left[\mathrm{M}^{*}\right]<[\mathrm{M}]$ ) o processo pode ser considerado pseudo- $1^{a}$ ordem, podendo ser estudado por este programa de simulação. $\mathrm{O}$ excímero mais conhecido é o pireno, ${ }^{5}$ que possui $\Delta \mathrm{G}=$ $-4 \mathrm{kcal} / \mathrm{mol}$ para a equação de formação acima, o que resulta em uma constante de equilíbrio $\mathrm{K}=\mathrm{k}_{12} / \mathrm{k}_{21}=855$. A Figura 7 mostra a simulação para este caso. Observa-se que o monômero $\mathbf{M}^{*}$ (no estado 1, curva N1) apresenta um decaimento exponencial simples, enquanto que o excímero (MM)* (no estado 2, curva N2) possui uma curva ascendente inicial que é devida à sua formação, seguida pelo decaimento posterior.

\section{CONSIDERAÇÕES FINAIS}

Neste trabalho, foram apresentadas várias simulações de importância no ensino de luminescência e de cinética de decaimento de estados excitados. O Método Monte Carlo permite ao aluno facilmente simular e visualizar os mecanismos de luminescência focando-se

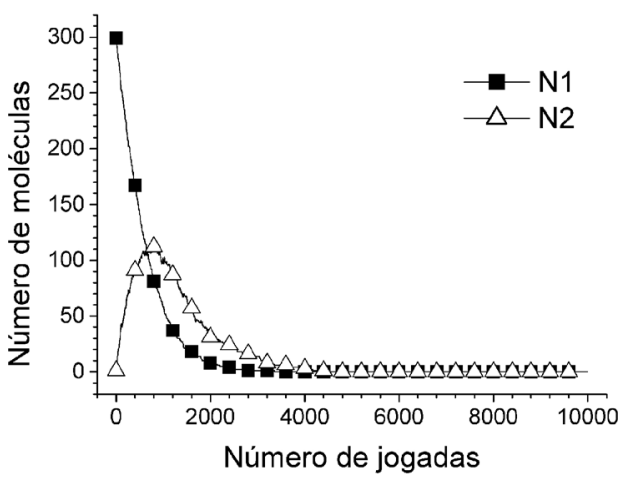

Figura 7. Curvas associadas ao sistema de dois estados excitados acoplados, exemplificando o processo de formação de excímeros. A simulação foi efetuada com 3 estados, 300 moléculas no estado 1 e probabilidades $k_{12}=1$, $k_{13}=0,005, k_{21}=0,002$ e $k_{23}=1$

nas probabilidades relativas das etapas em questão. O programa de simulação CINESTEX, ${ }^{6}$ utilizado neste trabalho, traz uma entrada de dados geral que permite que o aluno simule mecanismos mais complexos, com maior número de estados excitados e/ou com maior número de caminhos possíveis. Este programa foi feito em BASIC para compilador FreeBASIC, ${ }^{7}$ disponível para os sistemas operacionais Windows, Linux e DOS.

\section{REFERÊNCIAS}

1. Braslavsky, S. E.; Pure Appl. Chem. 2007, 79, 293.

2. Angelotti, W. F. D.; Fonseca, A. L.; Torres, G. B.; Custódio, R.; Quim. Nova 2008, 31, 433.

3. López-Castillo, A.; Souza Filho, J. C.; Quim. Nova 2007, 30, 1759.

4. Demas, J. N.; Excited State Lifetime Measurements, Academic Press: New York, 1983.

5. Turro, N. J.; Modern Molecular Photochemistry, University Science Books: Sausalito, 1991.

6. Novo, J. B. M.; CINESTEX; Programa para simulação Monte Carlo de cinética de decaimento de estados excitados; http://quimica.ufpr.br/ jbmnovo, acessada em Novembro 2009.

7. Victor, A.; FreeBASIC Compiler; Free, open source BASIC Compiler; http://www.freebasic.net/, http://sourceforge.net/projects/fbc/, acessadas em Novembro 2009; Debord, J.; FBMath; FreeBASIC Math library; http://sourceforge.net/projects/fbmath/, acessada em Novembro 2009. 